\title{
A New Simulation Technology Research for Missile Control System based on DSP
}

\author{
Bin Tian*, Jianqiao Yu, Yuesong Mei \\ Beijing Institute of Technology, Beijing, 100081, China \\ tianbin@bit.edu.cn
}

\begin{abstract}
Keywords: Control System; Rapid Prototyping; Hardware-in-the-loop Simulation
Abstract. Simulation technology is extremely important in the design process of control algorithm for missile weapon system. Different from digital simulation and traditional hardware-in-the-loop simulation, a rapid prototyping hardware-in-the-loop simulation technology is proposed which can confirm the control algorithm by implementing a prototype of the control system in the loop, making the simulation more reliable and effective. In the simulation process, a high-end computer as the HOST PC simulate the external physical environment and the internal physical characteristics of the missile while the control system running on a prototype DSP controller makes response to the real signals and gives real output. Through serial communication a high-speed real-time data exchange channel is built to complete the interaction of sensors and control commands. Simulation results for a certain type of missile weapon system show the rapid prototyping hardware-in-the-loop simulation technology is effective.
\end{abstract}

\section{INTRODUCTION}

The control algorithm is the core of a missile weapon system, and is the key to the success of flight test. Generally, control algorithm requires a certain number of flight tests to stereotypes. But only after the control algorithm is fully verified, the physical flight test can be conduct because of its high cost. The control algorithm usually can be fully verified after the processes of digital simulation, hardware-in-the-loop simulation and flight test etc ${ }^{[1]}$. The cost of the three stages is constantly increasing, the cost to fix the problems found in it also increasing. Because if we find mistakes no matter in which stage, we will must return back to the initial stage to start all over again. So the simulation approach of the control system plays an important role to control the development cycle and costs. Therefore to establish a reasonable simulation platform and simulation method to achieve the preliminary verification on the control system has become an important part in the design process of missile weapon system.

In this paper, a rapid prototyping hardware-in-the-loop simulation technology of missile control system is proposed which can be conduct in simple simulation conditions by implementing a prototype of the control system hardware in the simulation loop. Rapid prototyping hardware-inthe-loop simulation is far more fidelity than digital simulation, at the same time it is more simple and quick to implement than traditional hardware-in-the-loop simulation.

\section{RAPID PROTOTYPING HARDWARE-IN-THE-LOOP SIMULATION}

\section{Simulation technology}

Along with many advanced technologies applied to the missile weapon system, its development fee is more and more expensive, while many countries are facing the plight of budget crunch, simulation as one of the most basic technology, can save a lot of development fee, therefore the application and development of simulation technology are highly valued by the world military powers. At present, simulations for missile weapon system before the flight test include digital simulation and hardware-in-the-loop simulation.

The basis of digital simulation is describing the system by a set of equations and various mathematical languages. Ideal mathematical model should be composed of equations deduced directly by basic physical concept and engineering factors, while many complex events especially 
the control and guidance part must be emulated by empirical equations. On the basis of the mathematical model, digital simulation is implemented by using computer to computing the equations. The mathematical model for digital simulation requires nearly hundreds of flight tests to verify. Obviously, the digital simulation is mainly used for theoretical research and its simulation method is simple while the simulation results have large gaps compare with the real flight test.

Semi-physical simulation technology, also known as hardware-in-the-loop simulation, implement real objects as more as possible in the simulation system, to replace the corresponding parts of the mathematical model, making it more closer to actual situation and obtain more accurate information. This kind of simulation can simulate the dynamic characteristics of the real object on a computer by establishing the mathematical model and programming. It also requires corresponding sensors to measure the physical effects of the environment. Due to the real objects in the loop, the hardware-in-the-loop simulation system must run according to the real-time, so the hardware-in-theloop simulation system can be divided into the following sections: (1) the simulation computer system (dynamic model, program, data) and interface, (2) environment simulation equipment (angular motion simulators, target property simulator, target motion simulator, the load simulator etc.), and (3) real objects (sensors, control computer, actuator).

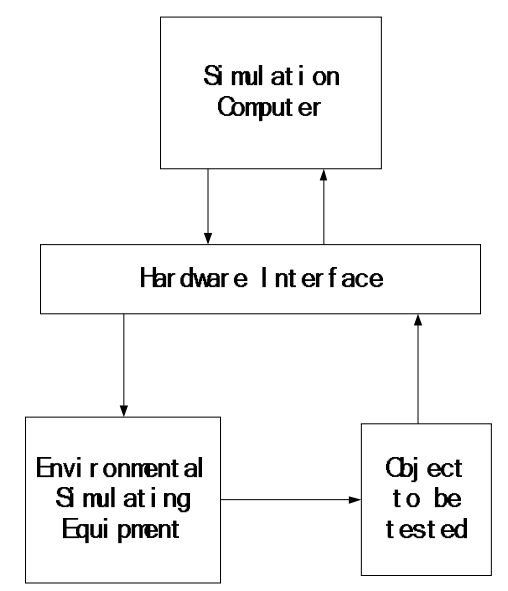

Figure1. The block diagram of traditional hardware-in-the-loop simulation

Hardware-in-the-loop simulation do not require flight test to provide the empirical equations and the coefficient data, so it improves the fidelity and validity of the simulation. Obviously, the hardware-in-the-loop simulation is more credible than digital simulation, while more complex and cost more at the same time ${ }^{[2]}$.

\section{Rapid prototyping hardware-in-the-loop simulation}

Rapid Prototyping means in the early stages of the product development quickly build the system model then conduct repeatedly non-real-time and real-time tests to verify the feasibility of the system hardware and software solutions, in the later stages via automatic code generation and code download on the target to complete the whole process of the product development.

Missile control system is a closed loop which missile as the controlled object, according to the relative flight conditions of missile and target, it can calculate the flight deviation of the actual position and the predetermined position to generate the control command, then control the missile changing its flight direction to complete the target interception. It is composed of flight control system, rudder, body dynamics and kinematics etc.

The design and development of missile control system rapid prototyping is to establish prototype platform, build mathematical model based on the principle of missile flight control system, then implement the process from model design to real-time product test and simulation. The rapid prototype of control system can participate in the whole process of the system design.

The process of control system rapid prototyping is to get the automatic code generation of the designed control algorithm, and download it directly to the hardware platform which can simulate 
the real control system. Then in a certain semi-physical simulation, get the operating result, and analyze each parameter index of control system to validate and correct the control algorithm ${ }^{[3,4]}$.

Rapid prototyping hardware-in-the-loop simulation proposed in this paper consulted the Rapid Prototyping method, transplant the control system model designed in digital simulation by code migration into the DSP hardware platform which is corresponding to the control system (implement in the actual generation environment of the hardware) and link in the simulation loop to implement real-time data exchange. Compared with the block diagram of traditional hardware-in-the-loop simulation system, the block diagram of rapid prototyping hardware-in-the-loop simulation system is shown in figure 2.

Rapid Prototyping hardware-in-the-loop simulation (RP Hardware in the Loop Simulation) can be conduct before and after the traditional hardware-in-the-loop simulation, if conduct before, it can confirm the hardware and software solutions to pave the way for traditional hardware-in-the-loop simulation, if conduct after, it can analysis and solve the problems found in the traditional hardware-in-the-loop simulation. It is a simulation technology which can approach the effects of the traditional hardware-in-the-loop simulation without enough conditions.

Compared with traditional hardware-in-the-loop simulation, rapid prototyping hardware-in-theloop simulation does not require special equipment and site environment for simulation. And compared to digital simulation, the prototype DSP controller used in rapid prototyping hardware-inthe-loop simulation is the real object used in traditional hardware-in-the-loop simulation, and there is real data exchange channel between the simulation computer and prototype DSP controller, have the characteristics of traditional hardware-in-the-loop simulation, approaching the effects of traditional hardware-in-the-loop simulation.

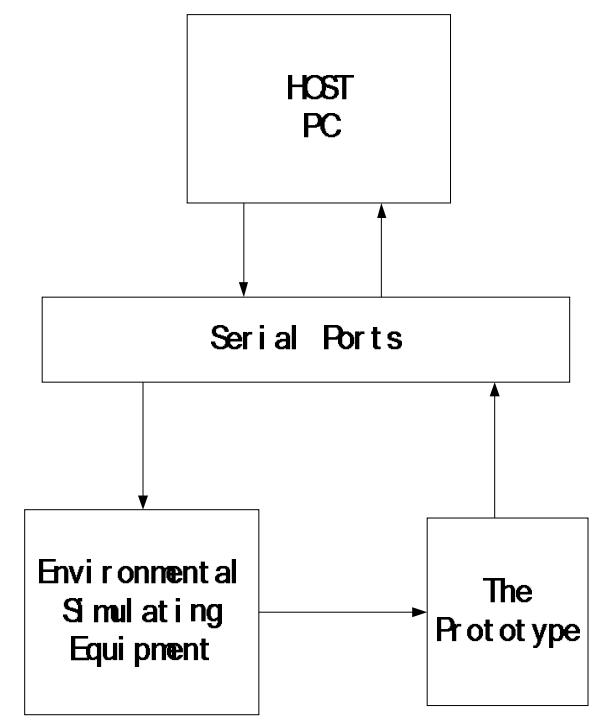

Figure2. The block diagram of rapid prototyping hardware-in-the-loop simulation system

\section{RAPID PROTOTYPING HARDWARE-IN-THE-LOOP SIMULATION APPLICATIONS}

Hardware-in-the-loop simulation with onboard computer/DSP processing unit participate in is the important part of hardware-in-the-loop simulation for the control system of missile. The block diagram of traditional hardware-in-the-loop simulation of control system is shown in figure 3. 


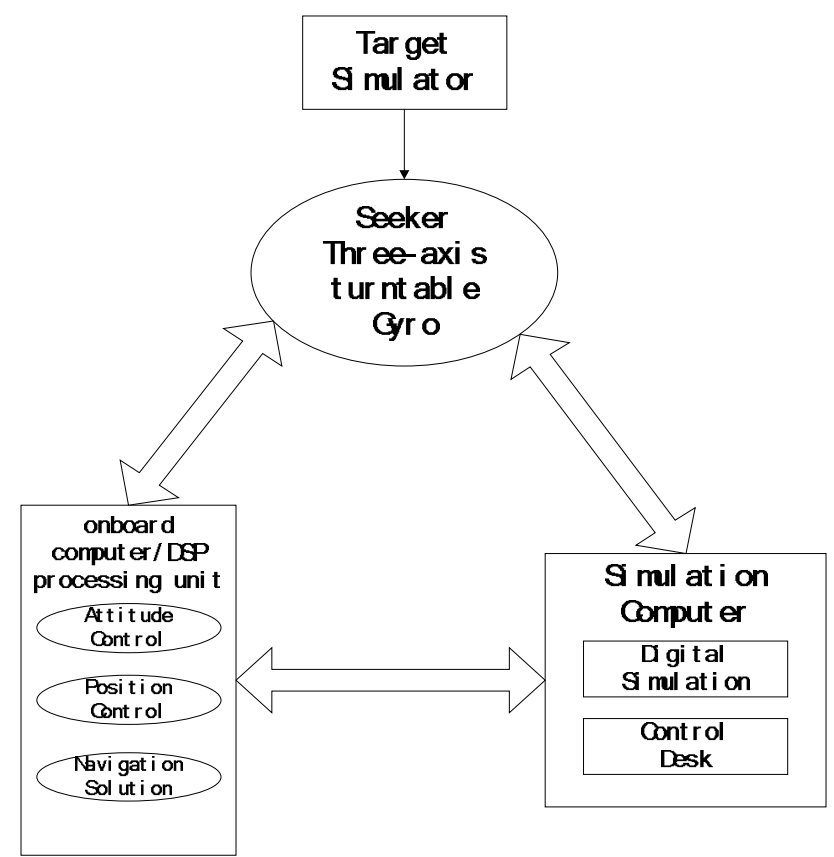

Figure3. The block diagram of traditional hardware-in-the-loop simulation of control system

The seeker, gyro and three-axis turntable are analog equipment of missile weapon system in hardware-in-the-loop simulation, which can transmits the attitude information, the gyro information and the attitude command to the onboard computer/DSP processing unit. Three-axis turntable can only simulate the attitude therefore the position is simulated by the corresponding digital model in the HOST PC. Onboard computer/DSP processing unit receive the attitude information, attitude command, position information, position command, gyro measurements to complete attitude control, position control, navigation solution and send control command to the HOST PC. The HOST PC running the missile body dynamics model, kinematics model, disturbance torques model, accelerometer model, actuator model etc.

In rapid prototyping hardware-in-the-loop simulation, a prototype DSP controller is connected to the CCS environment in an assistant computer composing the CCS/DSP processing unit to replace the onboard computer/ DSP processing unit, the seeker, three-axis turntable and other equipments are simulated by the digital simulation module in the VC environment. The block diagram of rapid prototyping hardware-in-the-loop simulation of control system is shown in figure 4.

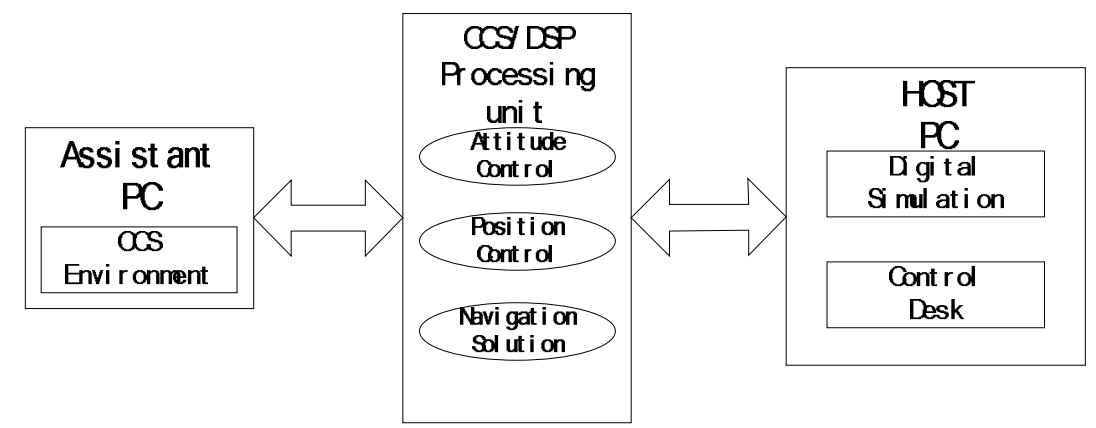

Figure4. The block diagram of rapid prototyping hardware-in-the-loop simulation of control system

In the rapid prototyping hardware-in-the-loop simulation with CCS/DSP processing unit participate in, CCS/DSP processing unit receive the attitude information, attitude command, position information, position command, gyro measurements, accelerometers measurements attitude control, position control, navigation solution and send control command to the HOST PC. The HOST PC running the missile body dynamics model, kinematics model, disturbance torques model, 
accelerometer model, gyro model, actuator model, error model etc. CCS / DSP processing unit and HOST PC make real-time information exchange.

\section{Example of rapid prototyping hardware-in-the-loop simulation System}

In this example, the control system uses TI (Texas Instrument) company's DSP as its information processor. TI's integrated development environment which named CCS (Code Composer Studio) provides a real-time online simulation function, which can connect to the target chip via an emulator to debug the target system ${ }^{[5]}$. The real-time channel includes two aspects: hardware channel and software channel. Hardware channel uses the XDS560 emulator to implement the physically connection between CCS environment and target system via the JTAG interface. Software channel apply TI's patented technology named RTDX (Real Time Data Exchange) which can provide a unified interface to complete the high-speed real-time data exchange between CCS environment and target system. Through the RTDX we can observe the running condition of the target system in real-time. The connection structure between target system and CCS environment is shown in figure 5.

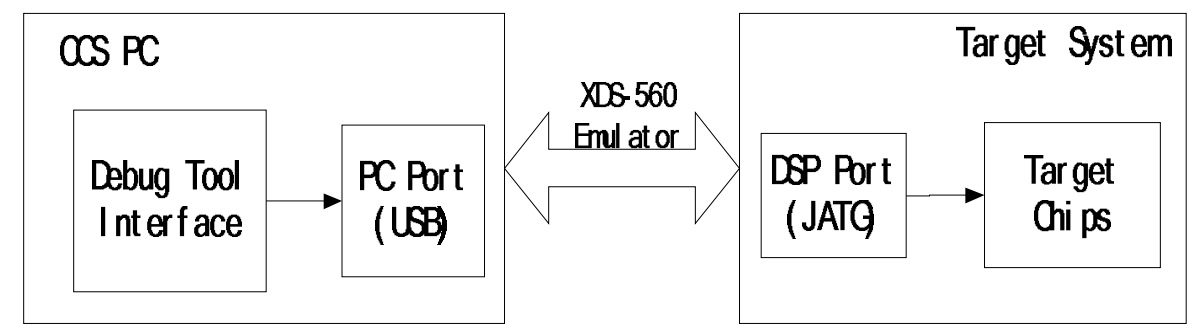

Figure5. The connection structure between target system and CCS environment

In rapid prototyping hardware-in-the-loop simulation, the $\mathrm{PC}$ with $\mathrm{CCS}$ environment as the Assistant PC load the control program of missile weapon system on the RAM of the CCS/DSP processing unit, the physical environment of missile weapon system is simulated by the corresponding dynamics model, kinematics model, torque model, gyro mode, accelerometer model, interface model in the HOST PC with VC environment.

CCS/DSP processing unit realize data exchange with the HOST PC through serial communication by using the SCI module. The hardware channel is RS232 interface and due to the DSP signal level is different from RS232 standard, so a MAX232 chip is applied for the level conversion. The software channel requires writing the control program both on DSP and PC at the same time ${ }^{[6]}$. On DSP, we use the CCS integrated environment to write the application program of SCI module. On PC programming, we write the program through the API provided by Windows to implement the operation of the serial port. The connection between HOST PC and CCS/DSP processing units is shown in figure 6.

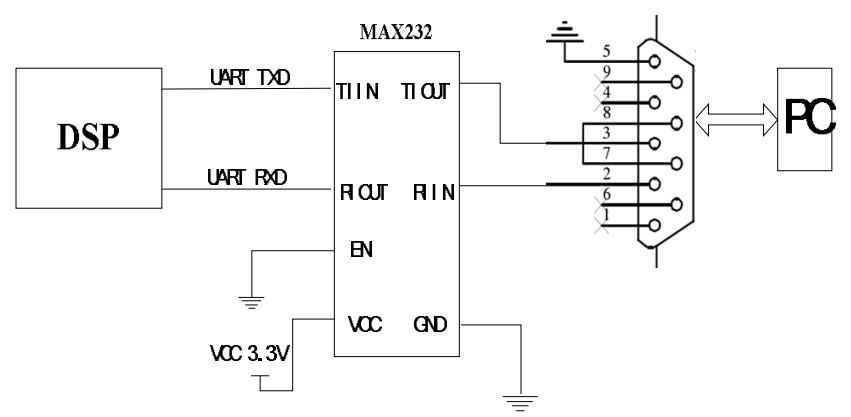

Figure6. The Serial Port Connection between HOST PC and PC CCS/DSP processing units

The whole simulation system flow chart is shown in figure 7. The HOST PC side is the process of missile trajectory simulation which simulated in advanced language development environment, 
the target system side is the process of control system which running on the CCS/DSP processing unit.

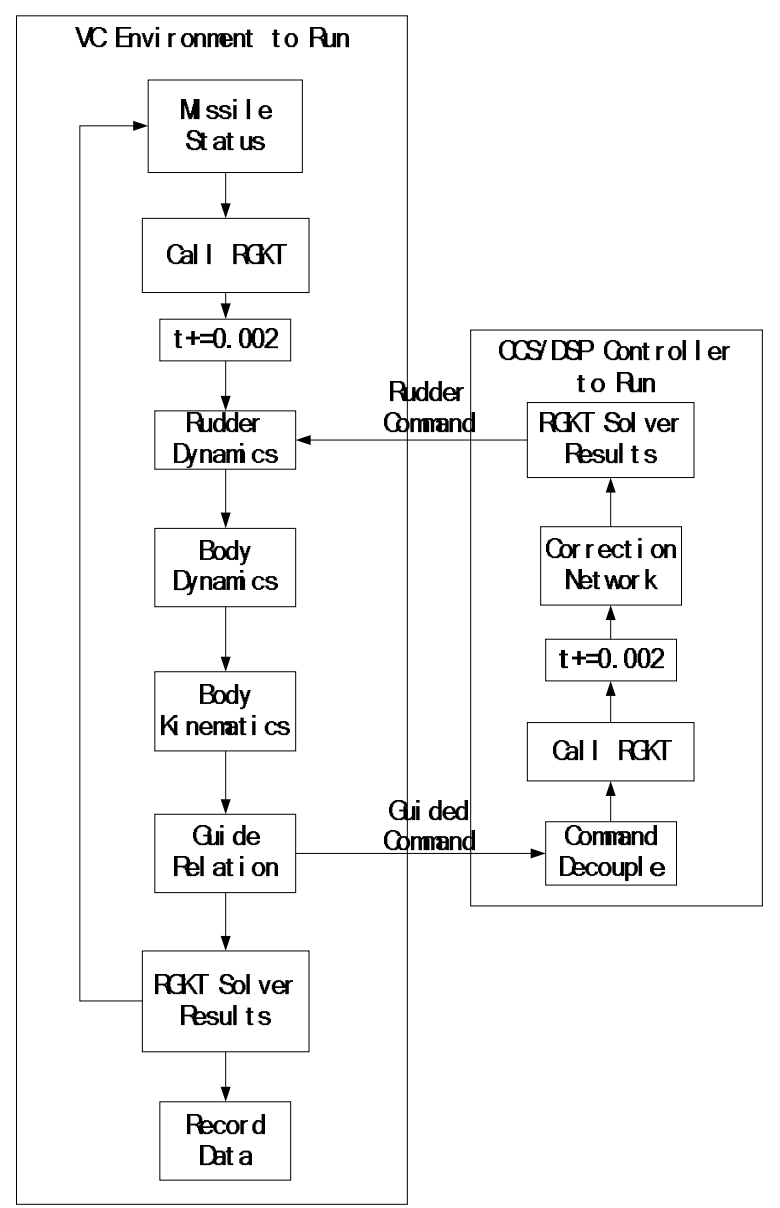

Figure7. The flow chart of simulation system

The simulation test result

In the simulation, the control system on the target is developed in the development platform of CCS version 3.3, the DSP use C28335 chip. In the missile trajectory simulation, missile space motion equations are solved by the fourth-order Runge-Kutta (RGKT) method. Due to the clock cycle of controller is $2 \mathrm{~ms}$ the solver step length is set to $2 \mathrm{~ms}$, triggering a periodic function per clock cycle to call the SCI interface to carry out exchange of control commands and rudder commands.

Under the same input conditions, here are the whole trajectory simulation test results of digital simulation and rapid prototyping hardware-in-the-loop simulation which are shown in figure 8-10. 


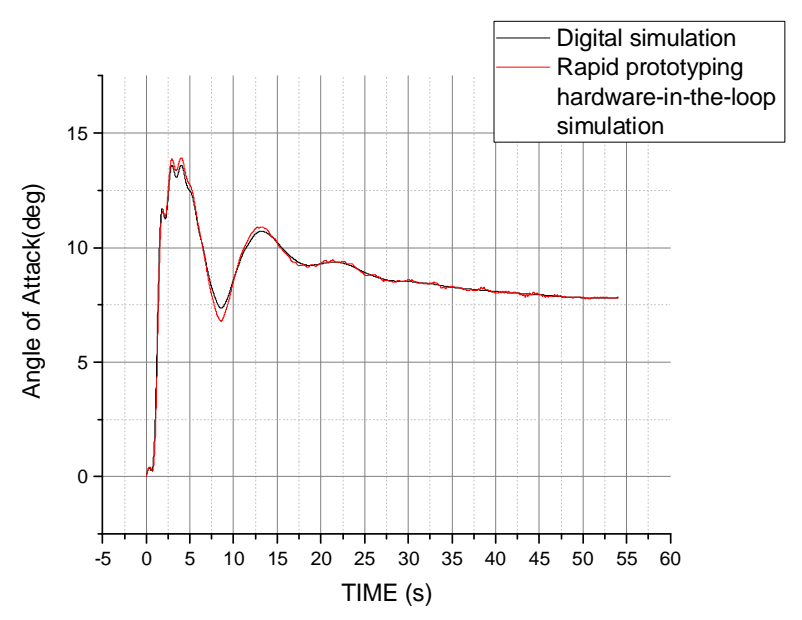

Figure8. Angle of attack curve comparison chart

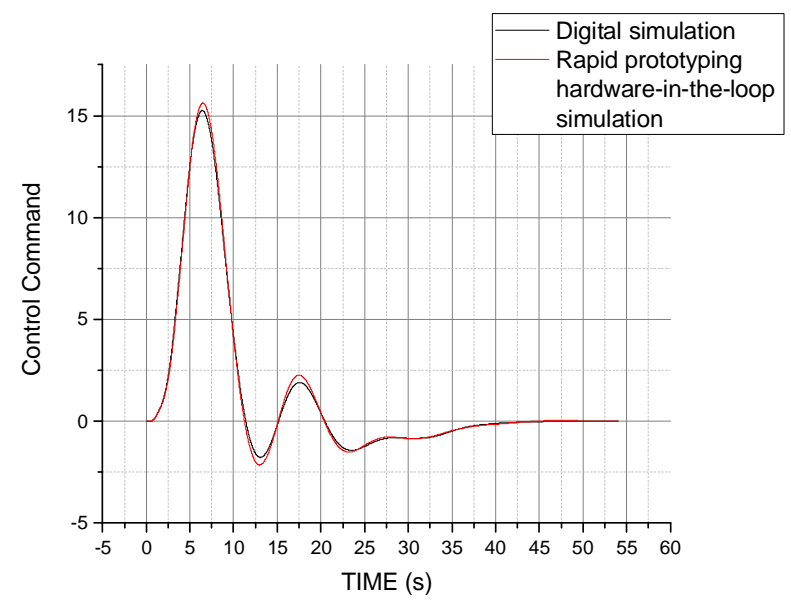

Figure9. Longitude control command curve comparison chart

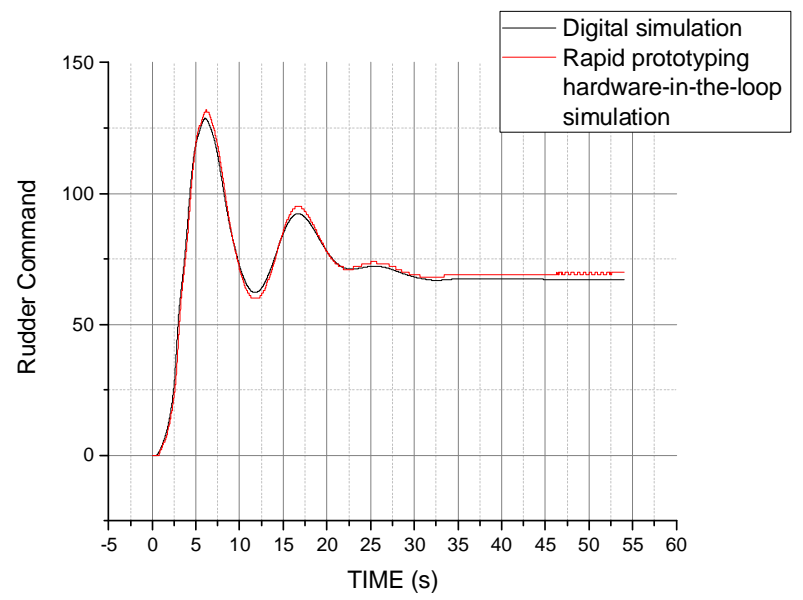

Figure10. Longitude rudder command curve comparison chart

Because there is a relatively sophisticated control algorithm in the simulation, the results of rapid prototyping hardware-in-the-loop simulation and digital simulation for missile control system built in $\mathrm{VC}$ environment are basically the same. In rapid prototyping hardware-in-the-loop simulation, 
due to the accuracy loss of data exchange caused by the incorporation of the real hardware interface, its simulation result curve has a slight concussion compared to digital simulation, the working conditions of the target control system are closer to the real environment.

\section{CONClusions}

This paper proposes the rapid prototyping hardware-in-the-loop simulation technology of control system for missile weapon system, which can be conduct when the traditional hardware-in-the-loop simulation conditions are not available, and under simple simulation conditions access the prototype object to the simulation loop, realize real-time interaction of the real object and simulation environment equipment, approaching the effects of traditional hardware-in-the-loop simulation. Therefore, rapid prototyping hardware-in-the-loop simulation as the intermediate and complementary aspects of digital simulation and traditional hardware-in-the-loop simulation can greatly reduce the development costs of missile weapon system and shorten the development cycle, improve research quality.

\section{REFERENCES}

[1] Garnell P. Guided Weapon Control System [M]. Beijing: Beijing Institute of Technology Press, 2004.

[2] Liu Xingtang. Missile Guidance and Control System Analysis, Design and Simulation [M]. Xian: Northwest Industrial University Press, 2006.(in Chinese)

[3] Ma Huimin, Zhang Huijun. Rapid Prototyping Technologies for Missile Guidance and Control System [J]. Electronics Optics\&Control, 2012(05): 78-81.(in Chinese)

[4] Du Runle, Wang Yonghai. A New Simulation Technology for Missiles Control System [J]. Missiles and Space Vehicles, 2010(02): 12-17.(in Chinese)

[5] Peng Qicong, Guan Qing, et al. DSP Integrated Development Environment [M]. Beijing: Electronic Industry Press, 2004. (in Chinese)

[6] Guo Weiwei, Ma Jiezhong, et al. Communication Research Between DSP and PC [J]. Micro Processers, 2008(04): 155-157.(in Chinese) 